\title{
Global Stability with Time-Delay in Network Congestion Control
}

\author{
Zhikui Wang and Fernando Paganini ${ }^{1}$
}

\begin{abstract}
This paper concerns the global stability of recently proposed laws for network congestion control. In earlier work it was shown that such laws were able to maintain local dynamic stability on networks of arbitrary delay, capacity and topology. In this paper we give conditions under which the stability can be shown to be global, focusing on the case of a single bottleneck network. The results include: a general boundedness result; a theorem on global asymptotic stability for the case of a single source, or many homogeneous ones, under quite sharp conditions; and a somewhat weaker asymptotic stability theorem for the heterogeneous source case.
\end{abstract}

\section{Introduction}

Congestion control in networks such as the Internet has been the subject of substantial analytical work in recent years. The starting point has been the explicit modeling of the feedback signal generated by congested links and communicated to sources; interpreting these signals as prices has allowed for the rate assignment to be cast as a utility optimization problem [7, 10], and to draw conclusions on the equilibrium structure. Also, dynamic models have been developed both for current TCP protocols and proposed variations. For a recent survey of this work, see [12].

Despite these advances, substantial difficulties remain in the understanding of these systems due to the combination of nonlinearity and time delay. Analytical results on, e.g. stability for large-scale networks typically handle only one of the above aspects. Focusing on nonlinearity but ignoring delays, Lyapunov proofs of stability of some protocols have been given in $[7,15,8]$; at the opposite end, $[4,13,16,18]$ provide stability results which include delay but work with linearized models. Results handling both nonlinearity and delay have been much more limited [3, 9]; this is not surprising considering that most advances in time-delay systems (see, e.g. [1]) appear to have occurred on the linear case. In contrast, nonlinear tools as those given in [2] involve

\footnotetext{
${ }^{1}$ Electrical Engineering Department, UCLA, Box 951594, Los Angeles, CA 90095-1594; Email: zkwang@ee.ucla.edu, paganini@ee.ucla.edu. Research supported by EPRI/DoD Grant WO-8333-06, and by the DARPA-ITO NMS program.
}

considerably more effort. For this reason, and perhaps inevitably, global stability results for network problems can at this time only be sought for smaller-scale cases involving for instance a single bottleneck link.

In this paper we focus on the recently developed control laws of [16], which have been shown to maintain local dynamic stability on networks of arbitrary delay, capacity and topology. We briefly review this work in Section 2. Next in Section 3 we focus on the single link case and explore its global properties with a variety of tools, including ideas of small-gain and integral quadratic constraints $[14,6]$, and also with LyapunovRazumikhin methods [2]. The first technique gives us less conservative conditions, but for a restrictive case: this is described in Section 3.1. The second method applies to the general case but under tighter assumptions, as shown in Section 3.3. In the process (Section 3.2) we derive a general boundedness result that may be useful in other contexts as well. Conclusions are given in Section 4.

\section{Control Laws with Local Scalable Stability}

In this section we define the congestion control problem under consideration and review the control laws of [16], which are proved to be locally stable for arbitrary networks and delays.

We are concerned with a system of $L$ communication links shared by a set of $S$ sources. The routing matrix $R$, of dimensions $L \times S$, is defined by

$$
R_{l i}=\left\{\begin{array}{ll}
1 & \text { if source } i \text { uses link } l \\
0 & \text { otherwise }
\end{array} .\right.
$$

Each source $i$ has an associated transmission rate $x_{i}(t)$; the set of transmission rates determines the aggregate flow $y_{l}(t)$ at each link, by the equation

$$
y_{l}(t)=\sum_{i} R_{l i} x_{i}\left(t-\tau_{l i}^{f}\right)
$$

in which the forward transmission delays $\tau_{l i}^{f}$ between sources and links are accounted for. We assume each link has a capacity $c_{l}$ in packets per second.

The next step is to model the feedback mechanism which communicates to sources the congestion information about the network. We associate with each 
link $l$ a congestion measure or price $p_{l}(t)[7,10]$, and assume sources have access to the aggregate price of all links in their route,

$$
q_{i}(t)=\sum_{l} R_{l i} p_{l}\left(t-\tau_{l i}^{b}\right) .
$$

Here again we allow for backward delays $\tau_{l i}^{b}$ in the feedback path from links to sources. As discussed in [12], this feedback model includes, to a good approximation, the mechanism present in existing protocols, with a different interpretation for price in different protocols. The total round-trip-time for the source thus satisfies

$$
\tau_{i}=\tau_{l i}^{f}+\tau_{l i}^{b}
$$

for every link in the source's path. This quantity can be estimated by sources in real time.

In this framework, a congestion control system is specified by choosing (i) how the links fix their prices based on link utilization; (ii) how the sources fix their rates based on their aggregate price. Ideally, these control laws should be such that upon a change in network conditions, the system is able to converge quickly to a new equilibrium with desirable properties. More specifically, in [16] the following requirements were laid down:

(i) At equilibrium, rates should not exceed a target capacity $\tilde{c}_{l}$ which is equal, or slightly below, the actual capacity, and the target should be matched at some bottleneck links.

(ii) Dynamically, the equilibrium point should be stable regardless of the network topology, parameters, or delay.

The design strategy adopted in [16] is to start from the linearized behavior around an equilibrium point $x_{0}, y_{0}, p_{0}, q_{0}$, and use the above requirements to guide the search for a local control law. Subsequently, the linearization is used to construct a global, nonlinear flow control.

Specifically, the following localized laws were considered: at the links, the price update law

$$
\delta \dot{p}_{l}=\frac{1}{\tilde{c}_{l}} \delta y_{l},
$$

and at the sources, the static control

$$
\delta x_{i}=-\frac{\alpha_{i} x_{0 i}}{M_{i} \tau_{i}} \delta q_{i} .
$$

Here $x_{0 i}$ is the equilibrium source rate, $\tau_{i}$ the RTT, $\alpha_{i}$ a constant, and $M_{i}$ a bound on the number of bottlenecks in source $i$ 's path. Under these local control laws, we have the following general result, which can be proved using tools of multivariable control. In it, $\bar{R}$ denotes the reduced routing matrix obtained by eliminating nonbottleneck links.
Theorem 1 ([16]). Suppose the matrix $\bar{R}$ is of full row rank, and that $\alpha_{i}<\frac{\pi}{2}$. Then link and source control laws that linearize around equilibrium as (3) and (4) give a locally stable system for arbitrary delays and link capacities.

The next step is to embed this linearized control in a global, nonlinear control scheme. At the links, since the gains are constant it is straightforward to implement the price dynamics as

$$
\dot{p}_{l}= \begin{cases}\frac{y_{l}-\tilde{c}_{l}}{\tilde{c}_{l}} & \text { if } p_{l}>0 \\ \max \left\{0, \frac{y_{l}-\tilde{c}_{l}}{\tilde{c}_{l}}\right\} & \text { if } p_{l}(t)=0 .\end{cases}
$$

Therefore prices integrate excess rate in a normalized way, and are saturated to be always non-negative. At equilibrium, bottlenecks with nonzero price will have $y_{l 0}=\tilde{c}_{l}$, and non-bottlenecks with $y_{l 0}<\tilde{c}_{l}$ will have zero price. With $\tilde{c}_{l}<c_{l}$ the equilibrium will have zero queues, as desired.

For the sources, [16] uses a static law $x_{i}=f_{i}\left(\tau_{i}, M_{i}, q_{i}\right)$, which makes this control a special case of those considered in [10]. Focusing on the dependence on the price $q_{i}$, the linearization requirement of (4) imposes that

$$
\frac{\partial x_{i}}{\partial q_{i}}=-\frac{\alpha_{i} x_{i}}{M_{i} \tau_{i}}
$$

for some $0<\alpha_{i}<\pi / 2$. Note also that around an equilibrium of (5), the real queues will be empty and therefore $\tau_{i}$ is the propagation delay. Assuming for now that $\alpha_{i}$ is constant, the above differential equation can be solved to give the static source control law

$$
x_{i}=x_{\max , i} e^{-\frac{\alpha_{i} q_{i}}{M_{i} \tau_{i}}}
$$

Here $x_{\max , i}$ is a maximum rate parameter, which can vary for each source, and can also depend on $M_{i}, \tau_{i}$ (but not on $q_{i}$ ). This exponential law for the source rates as a function of aggregate price can provide the desired linearization, together with the link control (5), and thus will satisfy the linear stability conditions in Theorem 1. The rest of this paper focuses on the nonlinear behavior of these control laws.

\section{Global Stability}

The work reviewed in the previous section establishes stability of the system defined by (5) and (7) for arbitrary networks, but only in a local, linearized sense. Nevertheless, flow simulation studies with simple networks have not as yet shown any examples where the convergence was not global as well. This leads us to explore in this paper an analytical proof of global stability. Due to the complexity of this task, we will only 


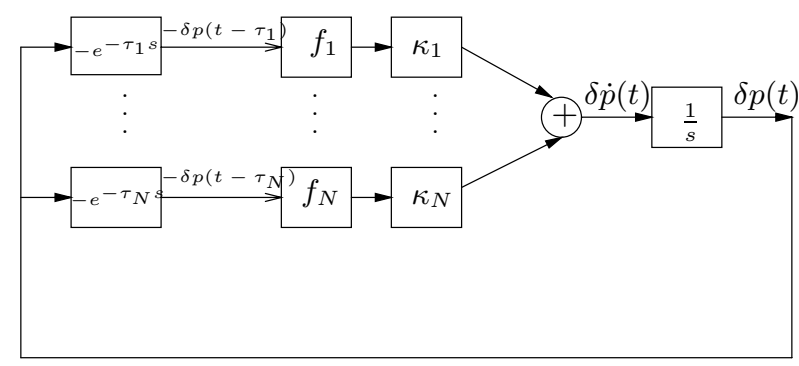

Figure 1: MultiSource-SingleLink Network

study the case of a single link accessed by $N$ heterogeneous sources. The closed loop dynamics are the following:

$$
\dot{p}(t)=\frac{1}{c}\left\{\sum_{i=1}^{N} x_{\max , i} e^{\frac{-\alpha p\left(t-\tau_{i}\right)}{\tau_{i}}}-c\right\}, \quad p(t) \geq 0,
$$

Here $p$ is the price of the single bottleneck, we have set $M_{i}=1$, and for simplicity we assume all sources have the same $\alpha$ parameter. We know local stability holds for $\alpha<\frac{\pi}{2}$; below we will give conditions on $\alpha$ (typically more conservative) where global stability can be proved.

At the equilibrium point $P_{0}$ of the price, we have

$$
\sum_{i=1}^{N} \frac{x_{\max , i}}{c} e^{\frac{-\alpha P_{0}}{\tau_{i}}}=1 .
$$

Then, for $\delta p(t)=p(t)-P_{0}$, we write the equivalent equations

$$
\delta \dot{p}(t)=\sum_{i=1}^{N} \kappa_{i}\left(e^{-\frac{\alpha}{\tau_{i}} \delta p\left(t-\tau_{i}\right)}-1\right), \quad \delta p(t) \geq-P_{0},
$$

where

$$
\kappa_{i}=\frac{x_{\max , i}}{c} e^{-\frac{\alpha}{\tau_{i}} P_{0}}, \quad \text { and } \quad \sum_{i=1}^{N} \kappa_{i}=1 .
$$

The system is shown in Figure 1, in which we define the nonlinear functions

$$
f_{i}(u)=e^{\frac{\alpha u}{\tau_{i}}}-1, \quad i=1,2, \ldots N
$$

Noticing that $\delta p \geq-P_{0}$, we have the constraint $u \leq P_{0}$. Therefore we limit the nonlinearity $f_{i}(u)$ to a conic sector

$$
f_{i} \in \sec \left[0, \beta_{i}\right], \quad i=1,2, \ldots, N
$$

with

$$
\beta_{i}=\frac{e^{\frac{\alpha}{\tau_{i}} P_{0}}-1}{P_{0}} .
$$

By this we mean that

$$
0 \leq f_{i}(u) u \leq \beta_{i} u^{2}, \quad i=1,2, \ldots, N
$$

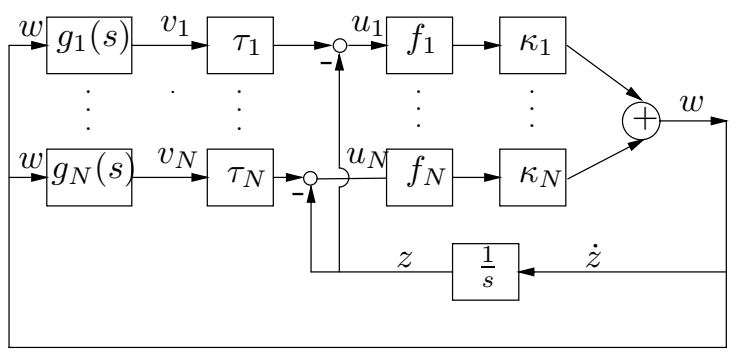

Figure 2: Equivalent System for Analysis

For some of the derivations to follow, it will also be convenient to rewrite the system in the equivalent interconnection of Figure 2. In it, we use the notation $z=\delta p, \dot{z}=w$, and

$$
v_{i}=\frac{1}{\tau_{i}} \int_{t-\tau_{i}}^{t} w(\sigma) d \sigma
$$

The equivalence of this system with the one in (10) follows from the identity

$$
-z\left(t-\tau_{i}\right)=-z(t)+\int_{t-\tau_{i}}^{t} \dot{z}(\sigma) d \sigma=-z(t)+\tau_{i} v_{i},
$$

which allows us to rewrite (10) as

$$
\dot{z}=\sum_{i} \kappa_{i} f_{i}\left(\tau_{i} v_{i}-z\right)
$$

as represented in Figure 2. Note also that by definition, the maps from $w$ to $v_{i}$ have transfer function

$$
g_{i}(s)=\frac{1-e^{-\tau_{i} s}}{\tau_{i} s}, \quad i=1,2, \ldots N
$$

The advantage of this configuration is that it "encapsulates" the delays in stable blocks with small gain: indeed, we have

$$
\left\|g_{i}(j \omega)\right\|_{\infty}=\sup _{\omega \in R}\left|\frac{1-e^{-j \omega \tau_{i}}}{j \omega \tau_{i}}\right|=1,
$$

so the $L_{2}$ gain from $w$ to $v_{i}$ is bounded by 1 .

\subsection{A stability theorem for the single source case}

We first present a stability proof under quite tight conditions on the gain parameter $\alpha$, but only applicable to the case of one source (or many sources with identical delay). In this case, it is found that the nonlinear (but delay-free!) system from $v$ to $w$ also has small gain. For this we use an argument which is adapted from [5]. We state the following result.

Theorem 2. For the configuration of Figure 2 when there is only one source, i.e.,

$$
\dot{z}(t)=f(\tau v(t)-z(t)),
$$




$$
\begin{gathered}
v(t)=\frac{1}{\tau} \int_{t-\tau}^{t} w(\sigma) d \sigma, \\
w(t)=\dot{z}(t),
\end{gathered}
$$

where $f(u) \in \sec [0, \beta]$. Suppose

$$
\beta \tau<1
$$

Then the equilibrium $z=0$ is globally asymptotically stable.

By global asymptotic stability for this system with time-delay, we mean that for initial conditions $z(0)=$ $z_{0}$ and $w(t)=w_{0}(t), \quad t \in[-\tau, 0]$, with $w_{0}(t) \in$ $L_{2}[-\tau, 0]$, we have

$$
z(t)^{2} \leq C_{1} z_{0}^{2}+C_{2}\left\|w_{0}\right\|^{2}
$$

for some positive constants $C_{1}, C_{2}$, and

$$
\lim _{t \rightarrow \infty} z(t)=0 \text {. }
$$

Proof: Multiplying by $\dot{z}$ in both sides of (17), we get

$$
\dot{z}^{2}=f(\tau v-z) \dot{z} .
$$

Noticing that $\dot{z}, f(\tau v-z)$, and $(\tau v-z)$ have all the same sign, we conclude from (14) that

$$
\dot{z}^{2} \leq \beta(\tau v-z) \dot{z}
$$

Integrating (22) in an interval $[0, T]$, we have, using Cauchy-Schwarz,

$$
\begin{aligned}
\int_{0}^{T} \dot{z}^{2}(t) d t & \leq \beta \tau \int_{0}^{T} v(t) \dot{z}(t) d t-\frac{1}{2}\left(z(T)^{2}-z_{0}^{2}\right) \beta \\
& \leq \beta \tau\|\dot{z}\|_{L_{2}[0, T]}\|v\|_{L_{2}[0, T]}-\frac{\beta}{2} z(T)^{2}+\frac{\beta}{2} z_{0}^{2} .
\end{aligned}
$$

Recall that $w=\dot{z}$, from (18) and (16), we know that $\|v\|_{L_{2}[0, T]} \leq\|w\|_{L_{2}[-\tau, T]}$, so

$$
\|w\|_{L_{2}[0, T]}^{2}-\frac{\beta}{2} z_{0}^{2} \leq \beta \tau\|w\|_{L_{2}[0, T]}\|w\|_{L_{2}[-\tau, T]}-\frac{\beta}{2} z(T)^{2} .
$$

Suppose now that we had $\lim _{T \rightarrow \infty}\|w\|_{L_{2}[0, T]}^{2}=\infty$. Then, dividing by $\|w\|_{L_{2}[0, T]}^{2}$ on both sides, and taking the limit we have

$$
1 \leq \beta \tau \lim _{T \rightarrow \infty} \frac{\|w\|_{L_{2}[-\tau, T]}}{\|w\|_{L_{2}[0, T]}}=\beta \tau,
$$

since the initial condition $\|w\|_{L_{2}[-\tau, 0]}$ is bounded. This contradicts the hypothesis $\beta \tau<1$. Therefore,

$$
w(t) \in L_{2}[0, \infty)
$$

Also, from (23),

$$
\begin{aligned}
z(T)^{2}-z_{0}^{2} & \leq \frac{2}{\beta}\left(\|w\|_{L_{2}[0, T]}\|w\|_{L_{2}[-\tau, T]}-\|w\|_{L_{2}[0, T]}^{2}\right) \\
& \leq \frac{2}{\beta}\left(\|w\|_{L_{2}[-\tau, T]}^{2}-\|w\|_{L_{2}[0, T]}^{2}\right) \\
& =\frac{2}{\beta}\|w\|_{L_{2}[-\tau, 0]}^{2}
\end{aligned}
$$

i.e., the state $z(t)$ is upper bounded as

$$
z(T)^{2} \leq z_{0}^{2}+\frac{2}{\beta}\left\|w_{0}\right\|_{L_{2}[-\tau, 0]}^{2}
$$

This establishes stability of the equilibrium point; it remains to show that $\lim _{t \rightarrow \infty} z(t)=0$.

To show $z(t)$ has a limit, integrate $(22)$ from $T$ to $T^{\prime}$ with $T^{\prime}>T>\tau$; similarily as above, we have

$$
z\left(T^{\prime}\right)^{2}-z(T)^{2} \leq \frac{2}{\beta}\|w\|_{L_{2}[T-\tau, T]} .
$$

Since $w(t) \in L_{2}[0, \infty]$, let $T \rightarrow \infty$, we have $\lim _{T \rightarrow \infty} z\left(T^{\prime}\right)^{2}-z(T)^{2}=0$. This establishes via the Cauchy criterion that

$$
\lim _{t \rightarrow \infty} z(t)=L, \quad \text { a finite number. }
$$

Moreover, from (18) we know that

$$
|v(t)| \leq \frac{1}{\sqrt{\tau}}\|w\|_{L_{2}[t-\tau, t]} \rightarrow 0 \text { as } t \rightarrow \infty .
$$

But then the right-hand side of (17) has limit $f(-L)$ as $t \rightarrow \infty$. Any nonzero $L$ would give a nonzero $\lim _{t \rightarrow \infty} \dot{z}(t)$, which is incompatible with $z(t)$ having a limit. Therefore we must have $L=0$.

Remark 1. In this single source case, the condition $\beta \tau<1$ can be translated into the original problem data. In fact, working with (9), (13) we arrive at the stability condition

$$
\alpha<\frac{\ln \left(\frac{x_{\max }}{c}\right)}{\frac{x_{\max }}{c}-1} .
$$

We assume here that $x_{\max }>c$, i.e. the link is a bottleneck. It is easy to see that the right hand side is less than 1 , so this condition is more conservative than our local stability test $\alpha<\pi / 2$.

If one wishes to extend this argument to the multiplesource case, the main difficulty is that the terms $\tau_{i} v_{i}-z$ in (15) need not have the same sign with $\dot{z}$, so the sector bound cannot readily be applied. We will therefore pursue an alternative route for this case.

\subsection{A boundedness result in the multi-source case}

We will show that for large $t$, and independently of any local stability conditions, the price can be bounded by an absolute constant. This will be useful in sharpening our sector bounds for a subsequent stability theorem.

Proposition 3. For the closed-loop system (8), there exists a finite time $t^{*}$ such that for all $t>t^{*}$, the price $p(t)$ is upper bounded by

$$
p(t) \leq P_{0}+\Delta P,
$$


where, with $\tau_{m}=\max _{i}\left(\tau_{i}\right)$

$$
\Delta P=\tau_{m}\left(\sum_{i=1}^{N} \frac{x_{\max , i}}{c}-1\right) .
$$

Proof: From (8) and (9), we know that, since $p(t) \geq 0$,

$$
\dot{p}(t) \leq \sum_{i=1}^{N} \frac{x_{\max , i}}{c}-1=\frac{\Delta P}{\tau_{m}},
$$

and

$$
\dot{p}(t)<0 \quad \text { if } \quad p(\sigma)>P_{0} \quad \text { for } \quad \sigma \in\left[t-\tau_{m}, t\right] .
$$

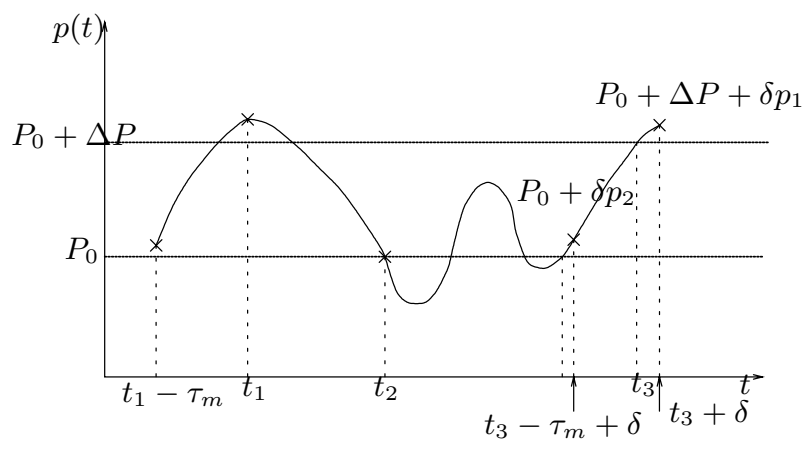

Figure 3: Upper bound of the state

Following those in Figure 3, assume that $p\left(t_{1}\right)>P_{0}+$ $\Delta P$ at some point $t_{1}>\tau_{m}$. We argue that it will go down to the range $\left[0, P_{0}\right]$. First, note the fact that

$$
p\left(t_{1}-\tau\right)>P_{0} \quad \text { for } \quad \tau \in\left[0, \tau_{m}\right] .
$$

Otherwise, $p\left(t_{1}\right)$ could not reach $P_{0}+\Delta P$ since the derivative is bounded by (32). Therefore, $\dot{p}(t)<0$ when $t \geq t_{1}$, and $p(t)$ will decrease till, at least, $t=t_{2}$ with $p\left(t_{2}\right)=P_{0}$, since $p\left(t-\tau_{i}\right)>P_{0}$ when $t \in\left[t_{1}, t_{2}\right)$.

Furthermore, we argue that the price $p(t)$ will not go above $P_{0}+\Delta P$ any more for $t>t_{2}$. Assume, instead, that it crossed this boundary at some point $t_{3}$. Then we would have $p\left(t_{3}\right)=P_{0}+\Delta P$ and $\dot{p}\left(t_{3}+\delta\right)>0$ for some $\delta>0$. However, similar to the above we have $p\left(t_{3}+\delta-\tau\right)>P_{0}$ for $\tau \in\left[0, \tau_{m}\right]$ so we conclude that $\dot{p}\left(t_{3}+\delta\right) \leq 0$, a contradiction. Therefore the state is upper bounded by $P_{0}+\Delta P$ for $t \geq t_{2}$.

We emphasize again that the bound does not require any restriction on parameters such as $\alpha$.

\subsection{A Razumikhin-type stability theorem for multiple sources}

We now present a second global stability theorem; this will apply to the multiple heterogeneous source case, however it will impose more conservative restrictions on the parameters.
Theorem 4. For the configuration of Figure 2, there exists a positive constant $\Theta$ such that for $\alpha<\Theta$, the origin of the system (10), i.e.,

$$
\dot{z}(t)=\sum_{i=1}^{N} \kappa_{i} f_{i}\left(-z\left(t-\tau_{i}\right)\right)
$$

is globally asymptotically stable.

Proof: We follow the example in [[2],P129] to show this theorem.

First it's assumed that the state variable $z(t)$ lies in the interval $\left[-P_{0}, \Delta P\right]$. This follows the earlier boundedness result if we consider sufficiently large times. This means the argument of the nonlinear functions $f_{i}(u)=e^{\frac{\alpha}{\tau_{i}} u}-1$ lies in the interval $\left[-\Delta P, P_{0}\right]$. In this region, we write the following bounds,

$$
\gamma_{i} \leq \frac{f_{i}(u)}{u} \leq \beta_{i}, \quad \text { and } \quad\left|\frac{\partial f_{i}(u)}{\partial u}\right| \leq \zeta_{i}
$$

where, for $i=1,2, \ldots, N, \beta_{i}$ is as in (13), and

$$
\gamma_{i}=\frac{1-e^{-\frac{\alpha \Delta P}{\tau_{i}}}}{\Delta P}, \quad \zeta_{i}=\frac{\alpha}{\tau_{i}} e^{\frac{\alpha P_{0}}{\tau_{i}}} .
$$

Consider the positive definite Lyapunov function $V(z(t))=\frac{1}{2} z^{2}(t)$, and the set of all $z(t)$ such that, for some $\eta>1$ and $\tau_{m}=\max _{i}\left(\tau_{i}\right), z^{2}(t-\sigma) \leq \eta^{2} z^{2}(t)$, for $\sigma \in\left[0,2 \tau_{m}\right]$. Then, following [2], we show the derivative of $V$ is decreasing under these assumptions. Specifically,

$\dot{V}(z(t))=z(t) \sum_{i=1}^{N} \kappa_{i} f_{i}\left(-z\left(t-\tau_{i}\right)\right)$

$=z(t) \sum_{i=1}^{N} \kappa_{i}\left[f_{i}(-z(t))-\left(f_{i}(-z(t))-f_{i}\left(-z\left(t-\tau_{i}\right)\right)\right)\right]$

$=z(t) \sum_{i=1}^{N} \kappa_{i}\left[f_{i}(-z(t))-\int_{t-\tau_{i}}^{t}\left(\frac{\partial f_{i}}{\partial z}(z(\sigma)) f_{i}\left(-z\left(\sigma-\tau_{i}\right)\right)\right) d \sigma\right]$

$\leq \sum_{i=1}^{N} \kappa_{i}\left[z(t) f_{i}(-z(t))+\zeta_{i} \beta_{i}|z(t)| \int_{t-\tau_{i}}^{t}\left|z\left(\sigma-\tau_{i}\right)\right| d \sigma\right]$

$\leq \sum_{i=1}^{N} \kappa_{i}\left[\frac{f_{i}(-z(t))}{z(t)}+\eta \zeta_{i} \beta_{i} \tau_{i}\right] z^{2}(t)$

$\leq-\sum_{i=1}^{N} \kappa_{i} \mu_{i} z^{2}(t)$

$=-\mu z^{2}(t)$

for some $\mu>0$, provided that, for $i=1,2, \ldots, N$,

$$
\frac{f_{i}(-z(t))}{z(t)}+\eta \zeta_{i} \beta_{i} \tau_{i} \leq-\mu_{i}<0 .
$$

If such conditions can be imposed, Theorem 4.2 in [2] implies that the origin of $z$ (i.e., $p(t)=P_{0}$ ), is globally asymptotically stable. 
From (36), a sufficient condition for (38) to hold could be, for $i=1,2, \ldots, N$,

$$
\beta_{i} \zeta_{i} \tau_{i}<\gamma_{i}
$$

For given parameters $N, c, x_{\max , i}$ and $\tau_{i}$, there must exist a constant $\Theta$ such that (39) is satisfied when $\alpha<$ $\Theta$. To see this, rewrite $(39)$ as

$$
h_{i}(\alpha):=\frac{\alpha^{2}}{1-e^{-\frac{\alpha \Delta P}{\tau_{i}}}}<\frac{\alpha P_{0}}{e^{\frac{\alpha P_{0}}{\tau_{i}}}\left(e^{\frac{\alpha P_{0}}{\tau_{i}}}-1\right) \Delta P} .
$$

Note that the product $\alpha P_{0}, \Delta P$, and $\tau_{i}$ are fixed when we decrease or increase the value of $\alpha$, leaving the remaining network parameters unchanged. $h_{i}(\alpha)$ is a strictly increasing function of $\alpha$ from 0 to $\infty$. Then, there exists a constant $\Theta_{i}$ such that the above condition is satisfied when $\alpha<\Theta_{i}$, although the dependence of this constant on the parameters is quite involved. We could then take the overall constant required to be $\Theta=\min _{i}\left(\Theta_{i}\right)$.

\subsection{Generalization to other source laws}

In the above theorems, the nonlinearity was handled via sector bounds, and therefore generalizations would in principle be available for any nonlinear source law that satisfied similar bounds. In particular, in recent work [17], motivated by fairness of equilibrium points, we have considered replacing the exponential law (7) by the power law

$$
x_{i}=\frac{\varphi\left(\tau_{i}, M_{i}\right)}{\left(M_{i} \tau_{i}+\beta_{i} q_{i}\right)^{\gamma_{i}}}, \quad \text { with } \quad \beta_{i}>0, \quad, \gamma_{i}>0,
$$

and shown that the system is locally stable when $\gamma_{i} \beta_{i}<$ $\pi / 2$.

In principle, the results of this paper would extend to such laws, the only difference being the specific formulas for the bounds which were given. For instance, the boundedness result of Section 3.2 would take the form

$$
p(t) \leq P_{0}+\max _{i}\left(\tau_{i}\right)\left(\frac{1}{c} \sum_{i=1}^{N} \frac{\varphi\left(\tau_{i}, M_{i}\right)}{\left(M_{i} \tau_{i}\right)^{\gamma_{i}}}-1\right),
$$

where $P_{0}$ is the equilibrium state.

\section{Conclusion}

We have obtained several conditions for the global asymptotic stability of the scalable controllers of [16], in the single link case. Moreover, we showed that the state is bounded without any limitation on the parameters. The conditions also extend to a more general family of nonlinear controllers, including those in [17].

A few topics remain open for the future research on the dynamic performance of the controllers. One issue is to make the stability condition less conservative, for instance, $\alpha<\pi / 2$, the same as that in the linear case, also suggested by empirical evidence. The other challenge is to extend the results to the multisourcemultilink case.

\section{References}

[1] L.Dugard, E.I.Verriest, "Stability and Control of Time-delay Systems", Springer-Verlag, New York, 1997.

[2] J. K. Hale, "Theory of Functional Differential Equations", Springer-Verlag, 1977.

[3] C.V.Hollot, Y. Chait, "Nonlinear stability analysis for a class of TCP/AQM schemes", Proc. IEEE CDC 2001, Dec. 2001.

[4] R. Johari, D. Tan, "End-to-End Congestion Control for the Internet: Delays and Stability",IEEE/ACM Transactions on Networking, Dec. 2001.

[5] U. Jonsson, A. Megretski, "The Zames-Falb IQC for Systems with Integrators, Automatica, March 2000.

[6] M. Jun, M.G.Safonov, "Stability analysis of a system with time-delayed states", Proc. of ACC, 2000.

[7] F. P. Kelly, A. Maulloo, D. Tan, "Rate control for communication networks: Shadow prices, proportional fairness, and stability", Jour. Oper. Res. Soc., 49(3), pp. 237252, 1998.

[8] S. Kunniyur, R. Srikant, "End-to-end congestion control schemes: utility functions, random losses and ECN marks", Proc. IEEE Infocom, 2000.

[9] S. Deb, R. Srikant, "Global Stability of Congested Controllers for the Internet", Coordinated Science Laboratory, UIUC, 2002. Anchorage: http://comm.csl.uiuc.edu/ srikant/pub.html.

[10] S. H. Low, D. E. Lapsley, "Optimization flow control - I: basic algorithm and convergence", IEEE/ACM Trans. on Networking, Vol 7(6) Dec 1999.

[11] S.H.Low, F.Paganini, J.Wang, S.Adlakha, J.C.Doyle, "Linear Stability of ICP/RED and a Scalable Control", Proceedings of the Allerton Conference, Monticello, IL, Oct., 2001.

[12] Steven H. Low, Fernando Paganini, John C. Doyle, "Internet congestion control: an analytical perspective", IEEE Control Systems Magazine, February 2002.

[13] L. Massoulie, "Stability of distributed congestion control with heterogeneous feedback delays," Technical report, Microsoft Research, Cambridge UK, TR 2000-111, 2000.

[14] A. Megretski, A. Rantzer, "System Analysis via Integral Quadratic Constraints", IEEE Transactions on Automatic Control, June, 1997

[15] F. Paganini, "On the stability of optimization-based flow control", Proc 2001 American Control Conference, 2001.

[16] F. Paganini, J. Doyle, S. Low, "Scalable Laws for Stable Network Congestion Control", Proceedings of the 2001 $C D C$, Orlando,FL, 2001.

[17] F. Paganini, S. Low, Z. Wang, S. Athuraliya, J.C. Doyle, "A new TCP congestion control with empty queues and scalable stability", submitted for publication.

[18] Glenn Vinnicombe, "A new TCP with guaranteed network stability," Tech. Rep., Cambridge Univiversity, preprint, June 2001. 